\title{
New Findings Supporting the Presence of Several Distinct Structures of Circumstellar Matter in $\beta$ Lyræ
}

\author{
P. Chadima ${ }^{1}$, P. Harmanec ${ }^{1,2}$, H. $\mathbf{A k}^{3}$, O. Demirçan ${ }^{4}$, S. Yang ${ }^{5}$, \\ P. Koubský ${ }^{2}$, P. Škoda ${ }^{2}$ M. Šlechta ${ }^{2}$, M. Wolf ${ }^{1}$, H. Božić ${ }^{6}$, \\ D. Ruždjak ${ }^{6}$ and D. Sudar 6 \\ ${ }^{1}$ Astronomical Institute of the Charles University, Faculty of Mathematics and Physics, \\ V Holešovičkách 2, CZ-180 00 Praha 8, Czech Republic \\ email: pavel.chadima@gmail.com, hec@sunstel.asu.cas.cz,wolf@cesnet.cz \\ ${ }^{2}$ Astronomical Institute of the Academy of Sciences, CZ-251 65 Ondřejov, Czech Republic \\ email: koubsky(skoda,slechta)@sunstel.asu.cas.cz \\ ${ }^{3}$ Department of Astronomy \& Space Sciences, Faculty of Arts \& Sciences, Erciyes University, \\ 38039 Kayseri, Turkey, email: hasan@physics.comu.edu.tr \\ ${ }^{4}$ Department of Physics, Faculty of Sciences and Arts, Çanakkale Onsekiz Mart University, \\ 17100 Çanakkale, Turkey, email: demircan@comu.edu.tr \\ ${ }^{5}$ Department of Physics and Astronomy, University of Victoria, P.O. Box 3055 STN CSC, \\ Victoria, B.C., Canada V8W 3P6, email: yang@uvastro.phys.uvic.ca \\ ${ }^{6}$ Hvar Observatory, Faculty of Geodesy, Kačićeva 26, 10000 Zagreb, Croatia \\ email: hbozic(dsudar,rdomagoj)@geof.hr
}

\begin{abstract}
. (1) 52 photographic and 651 electronic spectra were disentangled using the program KOREL. This led to the detection of a number of weak absorption lines originating in the atmosphere of the accretion disk. So far, the detection of this disk spectrum was only reported for the Si 6347 and $6371 \AA$ doublet. (2) The basic spectrophotometric quantities of 15 absorption lines of the primary were measured and corrected for the orbital light changes in order to eliminate the contribution of the secondary light to the observed continuum level. After the correction, a significant phase dependency of the spectral-line characteristics near the primary eclipse was detected. In all probability, another so far unknown absorption-line spectrum was thus found. This spectrum may be due to additional absorption of light of the primary in one of the jet-like structures and in the spherical gas envelope surrounding the accretion disk. Both these structures are seen projected against the primary during its eclipse.
\end{abstract}

Keywords. stars: individual ( $\beta$ Lyr), stars: variables: eclipsing, binaries: close, circumstellar matter, accretion disks

\section{Introduction}

$\beta$ Lyr is eclipsing and interacting binary which is at the stage of high mass-transfer rate between the components. Circumstellar matter most probably consists of a geometrically and optically thick accretion disk which entirely hides the light from the secondary, jet-like structures perpendicular to the orbital plane and a scattering gas envelope surrounding the disk around the secondary. (See Harmanec 2002 and references therein for the full history of investigation of $\beta$ Lyr).

In the present study, we used two sets of spectra - 52 "blue" digitized photographic spectra secured at the Ondřejov Observatory, Czech Republic and 651 "red" electronic spectra, secured at Ondřejov and at the Dominion Astronomical Observatory, Canada. 

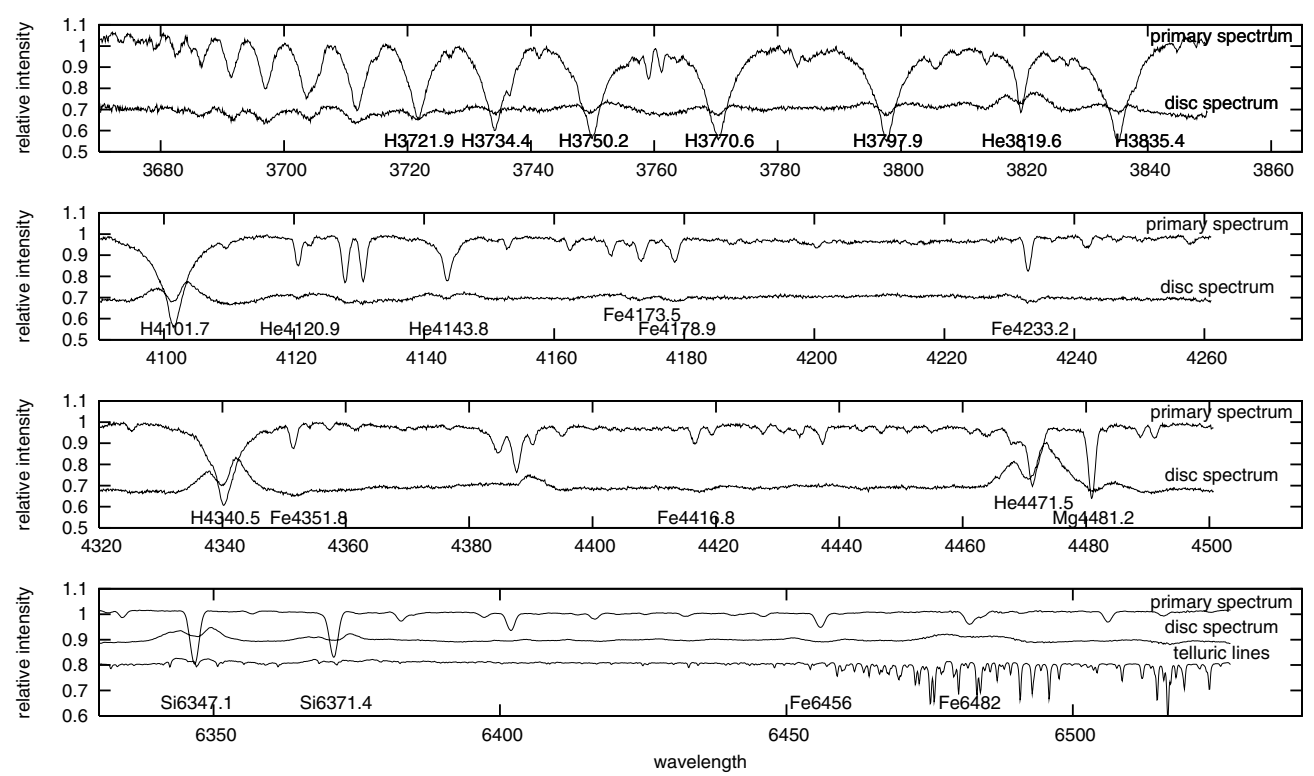

Figure 1. Disentangling of four spectral regions. Emission lines, seen in the disk spectra, originate in fact in the jet-like structures but our attempt to disentangle them separately failed.

\section{Disentangling of spectra}

Four selected spectral regions were disentangled using the KOREL program (Hadrava 2004). The results of the disentangling are displayed in Figure 1. In each panel, the primary spectrum is shown on the top and the disk spectrum below it. For electronic spectra longward of about $6300 \AA$, it was also necessary to include the telluric lines into the solution.

Note that the disentangling procedure led to the discovery of a number of weak absorption lines originating in the pseudophotosphere of the accretion disk. This way, we obtained a rich line spectrum of the disk, not limited to the previously known Si II doublet. Moreover, the radial velocities from the accretion-disk spectra define well a sinusoidal curve in antiphase to that of the primary.

\section{Spectrophotometry of absorption lines}

Central intensity (CI) and equivalent width (EW) of 15 stronger absorption lines were measured and further proceeded. These lines belong to the primary but their measurements refer to the common continuum of both, the primary and the accretion disk. The measured quantities must therefore be corrected to the continuum of the primary star only. The correction equations are as follows

$$
C I_{\text {corr }}=1-\frac{F_{p}(\lambda, f)+F_{d}(\lambda, f)}{F_{p}(\lambda, f)}\left(1-C I_{o b s}\right), \quad E W_{c o r r}=\frac{F_{p}(\lambda, f)+F_{d}(\lambda, f)}{F_{p}(\lambda, f)} E W_{o b s} .
$$

Subscript obs means observational and subscript corr corrected values. $F_{p}$ and $F_{d}$ denote the relative monochromatic fluxes of the primary and of the accretion disk at given wavelength $\lambda$ and orbital phase $f$. To evaluate this correction factor, it is necessary to use a light-curve solution which takes into account the presence of the thick accretion disk. To this end, we used the latest version of the BINSYN program (Linnell 2000).

In Figure 2, there are the phase plots of CI and EW of Si II 6371 line. Note a very pronounced line strengthening in the phases around the primary eclipse. The same phase 

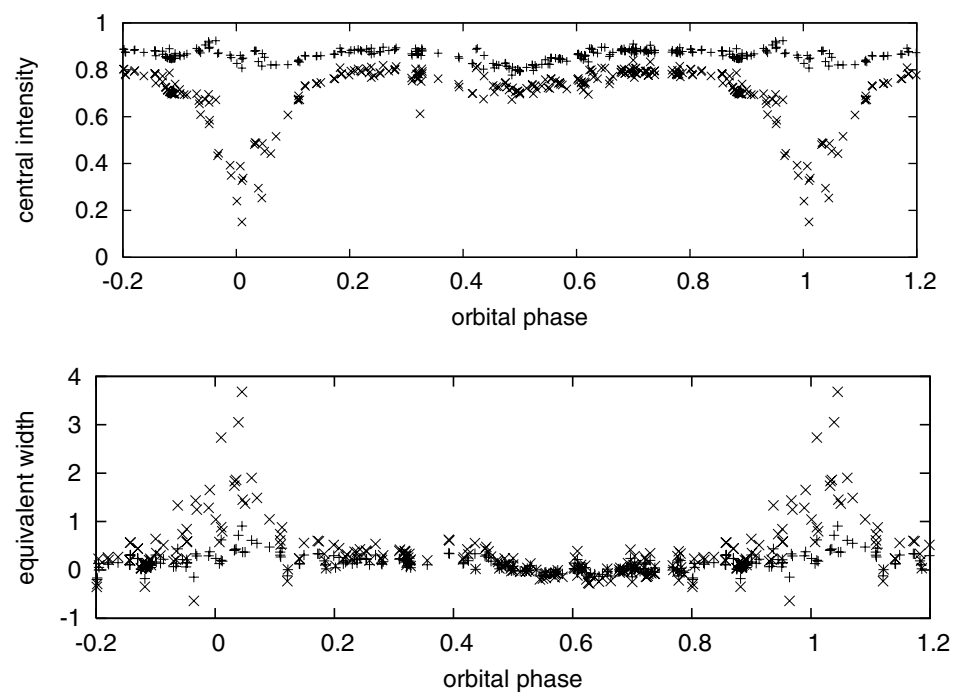

Figure 2. Phase dependency of central intensity and equivalent width of Si II 6371 line. Observed values are shown as + , corrected values as $\times$.

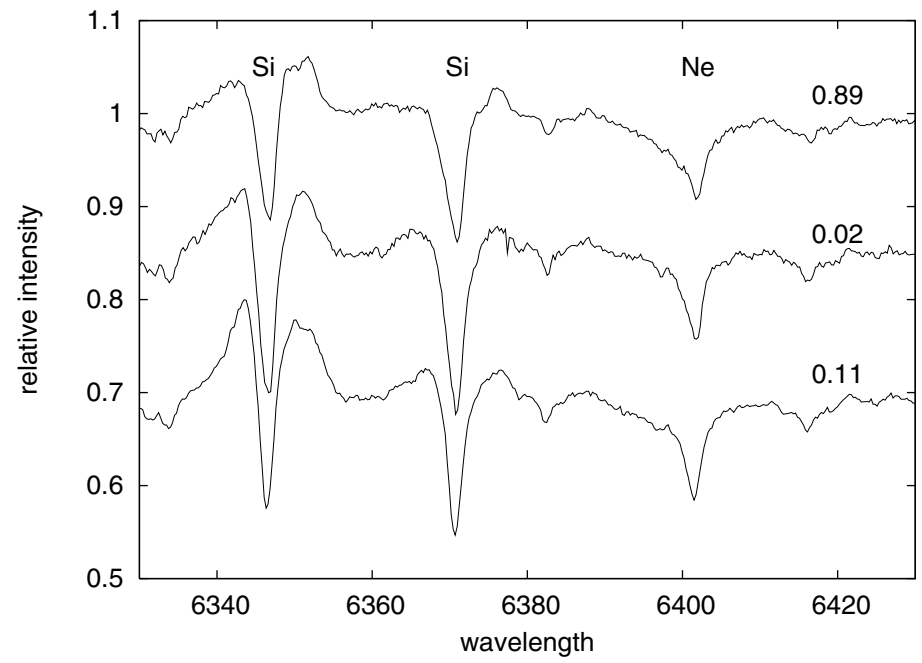

Figure 3. Three spectra around the primary eclipse. Orbital phases are given on the right side.

dependence was found also for all other investigated lines. These changes are too large to be caused by physical or geometrical properties of the primary. This effect can be also demonstrated in Figure 3. Note that the absorption lines in phases near the primary mid-eclipse are deeper which contradicts the fact that most of the primary surface is hidden from the view in this interval of phases.

It seems that the only plausible explanation is the following one: During the eclipse, there is always a certain part of the primary which remains uneclipsed by the disk and the light from it passes through both, the gaseous envelope and one of the jets which leads to formation of additional absorption lines. It means that there is another absorption-line spectrum observable only in the phases of the primary eclipse. 


\section{Conclusion}

Both analyses led to the discovery of a new set of spectral lines - the accretion disk spectrum and the "shell" spectrum originating at the jet and/or the scattering envelope above the disk, providing additional support to the current model of $\beta$ Lyr.

\section{Acknowledgements}

We acknowledge the use of the programs SPEFO, BINSYN and KOREL, made available by their authors J. Horn \& J. Krpata, A.P. Linnell \& I. Hubeny and P. Hadrava. Our special thanks go to A.P. Linnell for his patient advice concerning the proper use of BINSYN. Some of the Ondřejov spectra were obtained by P. Hadrava, D. Holmgren, J. Horn, J. Kubát, V. Šimon and S. Štefl. We profitted from the use of electronic bibliography maintained by the NASA/ADS system. Czech authors were supported from the research plans J13/98: 113200004 of Ministry of Education, Youth and Sports and AV $0 Z 1$ 003909, from project K2043105 of the Academy of Sciences of the Czech Republic and from the grants GA ČR 205/02/0788 and 205/06/0304 of the Czech Science Foundation. Turkish authors were partly supported by the Research Council of Turkey and the Research Foundation of Çanakkale University. Collaboration between Turkish and Czech authors was facilitated thanks to a NATO collaborative linkage grant PST.CLG.979343.

\section{References}

Hadrava, P. 2004, Publications of Astron. Inst. of Academy of Sciences of the Czech Rep. 92, 15 Harmanec, P. 2002, Astronomische Nachrichten 323, 87

Linnell, A.P. 2000, MNRAS 319, 255 